\title{
Plant Leaf Identification Using Multi-scale Fractal Dimension
}

\author{
André R. Backes ${ }^{1}$ and Odemir M. Bruno ${ }^{2}$ \\ 1 Instituto de Ciências Matemáticas e de Computação (ICMC) \\ Universidade de São Paulo (USP) \\ Avenida do Trabalhador São-carlense, 400 \\ 13560-970 São Carlos SP Brazil \\ backes@icmc.usp.br \\ 2 Instituto de Física de São Carlos (IFSC) \\ Universidade de São Paulo (USP) \\ Avenida do Trabalhador São-carlense, 400 \\ 13560-970 São Carlos SP Brazil \\ bruno@ifsc.usp.br
}

\begin{abstract}
Taxonomical classification of plants is a very complex and time-consuming task. This is mostly due to the great biodiversity of species and the fact of most measures extracted from plants are traditionally performed manually. This paper presents a novel approach to plant identification based on leaf texture. Initially, the texture is modelled as a surface, so complexity analysis using Multi-scale fractal dimension can be performed over the generated surface, resulting in a feature vector which represents texture complexity in terms of the spatial scale. Yielded results show the potential of the approach, which overcomes traditional texture analysis methods, such as Co-occurrence matrices, Gabor filters and Fourier descriptors.
\end{abstract}

Keywords: plant identification, complexity, multi-scale fractal dimension, texture analysis.

\section{Introduction}

Many scientific problems related to biodiversity, ecology and pharmacology depend on plant identification. In Biology, this task involves the analysis of many organs, such as flowers, seeds, leaves and woody parts $[12$. However, some characteristics depend on plant age or environment, what makes this task difficult to accomplish (e.g., flowers). Otherwise, leaves can be easily collected from most plants, including fossils or rare plants, so becoming a interesting source of information 3 .

Plant leaf identification is a difficult task due to the wide pattern variation of its fundamental features. Variations in size, color, texture and shape, are found in leaves collected from a same plant (a response to different levels of maturity and sun exposure) or in samples from different plants (soil influence, climate or even environment). 
Texture is one of the most important visual attributes in images. It allows to describe the surface of a leaf in terms of the distribution of pixels over a region. Literature presents a wide number of approaches to describe texture patterns: second-order statistics [45], spectral analysis 6/7/891011/2] and wavelet packets [13 14. However, different of human-made textures, which present a detectable quasi-periodic structure, natural textures (such as, leaves texture) present a random but persistent pattern that results in a cloud like texture appearance [1516].

An interesting alternative to these approaches is methods based on complexity analysis, such as fractal dimension. Fractals are objects which the dimension takes non-integer values (non-Euclidean geometry). Many natural phenomena and surfaces (e.g.,coastlines, brick, skin, rocks, etc) can be modelled using the fractal theory. Thus, by modelling the leaf texture as a surface it is possible to estimate its fractal dimension and, as a consequence, to describe its texture pattern in terms of space occupation and self-similarity [17 18 19].

This paper starts describing how texture analysis is performed by complexity (Section 2). A multi-scale approach for texture characterization is presented in Section 3. In Section 4, we describe how the leaf texture database was composed. Statistical analysis is employed to evaluate our approach. The results are presented and discussed in Section [5 while Section 6 concludes this paper.

\section{Texture Analysis Based on Fractal Dimension}

The fractal dimension has been widely used in literature to characterize both real and abstract objects in terms of complexity. While the topological dimension is defined by an integer value, which describes the number of dimensions where an object is inserted, the fractal dimension uses fractional values to describe an object in terms of space occupation and self-similarity $2021|22| 23|24| 17 \mid 18]$.

Over the years, many approaches have been proposed to compute the fractal dimension. Bouligand-Minkowski method [18,21|25] has emerged as one of the most accurate methods and very sensitive to structural changes of the object. It is based on the study of the influence area of an object computed from its dilation. Let the gray-scale texture under analysis be represented in terms of the set $S \in R^{3}$ of the Cartesian coordinates. Each element $s \in S, s=(y, x, z)$, is defined by the pixel coordinates $(y$ and $x$ ) in the original texture and the intensity $z$ at this point. Let $S(r)$ be the dilation of $S$ by a sphere of radius $r$ :

$$
S(r)=\left\{s^{\prime} \in R^{3}|\exists s \in S:| s-s^{\prime} \mid \leq r\right\},
$$

where $p^{\prime}=\left(x^{\prime}, y^{\prime}, z^{\prime}\right)$ is a point in $R^{3}$ whose distance from $p=(x, y, z)$ is smaller or equal to $r$ (Figure 1).

The Bouligand-Minkowski fractal dimension $D$ is defined as

$$
D=3-\lim _{r \rightarrow 0} \frac{\log S(r)}{\log (r)},
$$

where $D$ is a number within $[0 ; 3]$ (i.e. considering a three-dimensional space). 


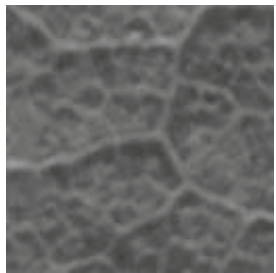

(a)

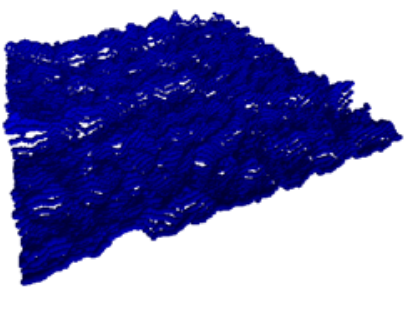

(b)

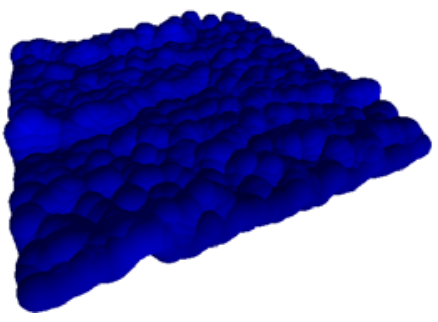

Fig. 1. (a) Original Texture; (b) Texture modelled as Cartesian coordinates; (c) Dilation using $r=10$

\section{Multi-scale Fractal Dimension}

Natural objects are not real fractals. They may present an infinite degree of details along the scales, although its self-similarity along these scales is not preserved. Usually, fractal dimension $D$ is computed as the angular coefficient of the logarithm curve of the volume $(S(r))$ in terms of dilating radius, using linear interpolation. However, the logarithm curve computed presents more details than can be expressed by a single numeric value and, eventually, the fractal dimension $D$ is not enough to represent all complexity of an object.

In order to provide a better description of objects in terms of its complexity, the Multi-Scale Fractal Dimension has been proposed [26/27/21]. This approach involves taking into the infinitesimal limit the linear interpolation by using the derivative, so achieving a function capable to express the complexity of an object in terms of the spatial scale (Figure 2). This function provides a more effective discrimination of the object, and it is defined as:

$$
D(r)=3-\frac{d \log S(r)}{d r},
$$

where $D(r)$ represents the complexity of the object at scale $r$.

\section{Experiments}

Experiments were conducted considering a texture leaf database built using 10 leaves species from Brazilian flora. A total of 3 leaves samples was manually collected for each species considered. Each leaf was washed to remove impurities that could act as noise in the texture pattern. The digitalization process was carried out by a scanner using a 1200dpi (dots per inch) resolution and leaves were oriented according to the central axis (line that connects the basal and apical ends) in a vertical position during this process.

From each digitalized leaf, a total of 5 texture windows of $128 \times 128$ pixels of size were extracted, totalising a database with 150 texture samples divided into 


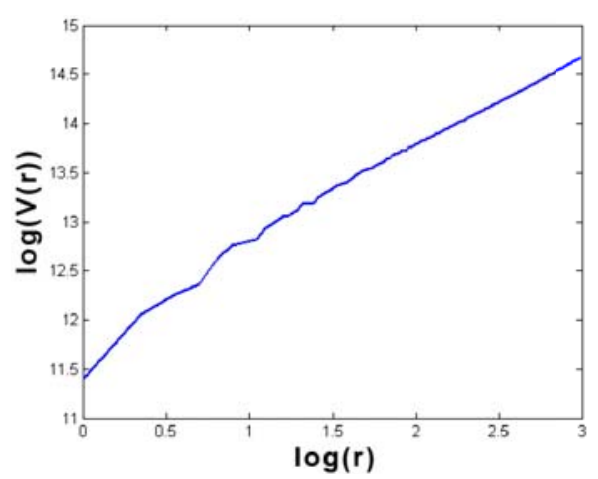

(a)

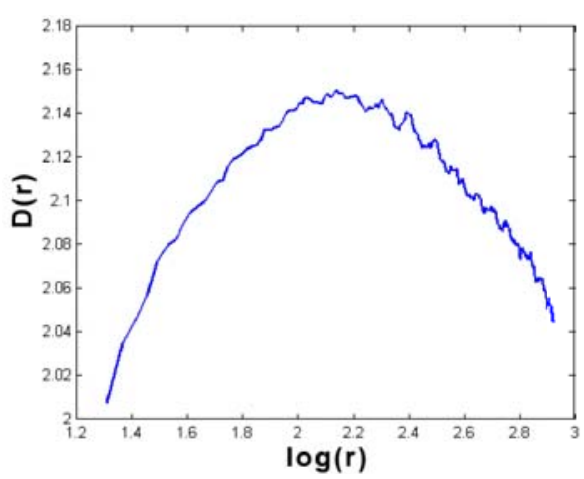

(b)

Fig. 2. (a) Log-log curve; (b) Multi-scale Fractal Dimension

10 classes (Figure 3). All color information was discarded, i.e., only the grayscale information from the texture pattern was considered. It is important to emphasize that one single leaf may present a great variability of texture patterns (Figure 4). This variation in texture patterns can be caused by different factors, such as fungus, plague, injuries or even differences in the amount of light received by the leave during its lifetime. Thus, the selection of texture windows was guided to avoid such texture patterns that do not characterize the real texture of that leaf specie.
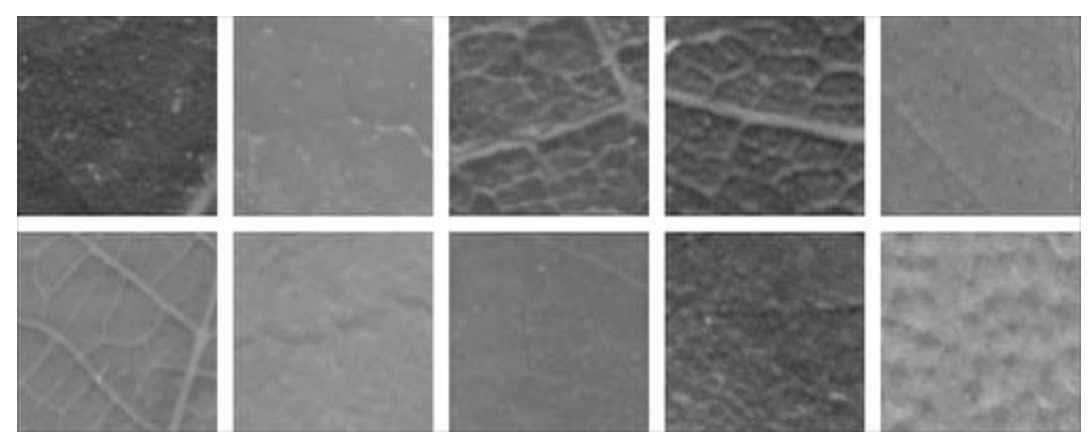

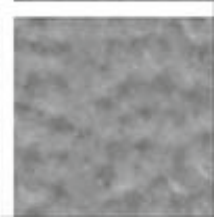

Fig. 3. Example of each texture class employed in the experiment

Evaluation of the Multi-scale curve was carried out using Linear Discriminant Analysis (LDA), a supervised statistical classification method 2829]. LDA searches a linear combination of the descriptors (independent variables) that results in its class (dependent variable). Its main goal is to find a linear combination that minimizes the intra-classes variance while maximizes the inter-classes variance. The leave-one-out cross-validation strategy was also employed over the LDA. 

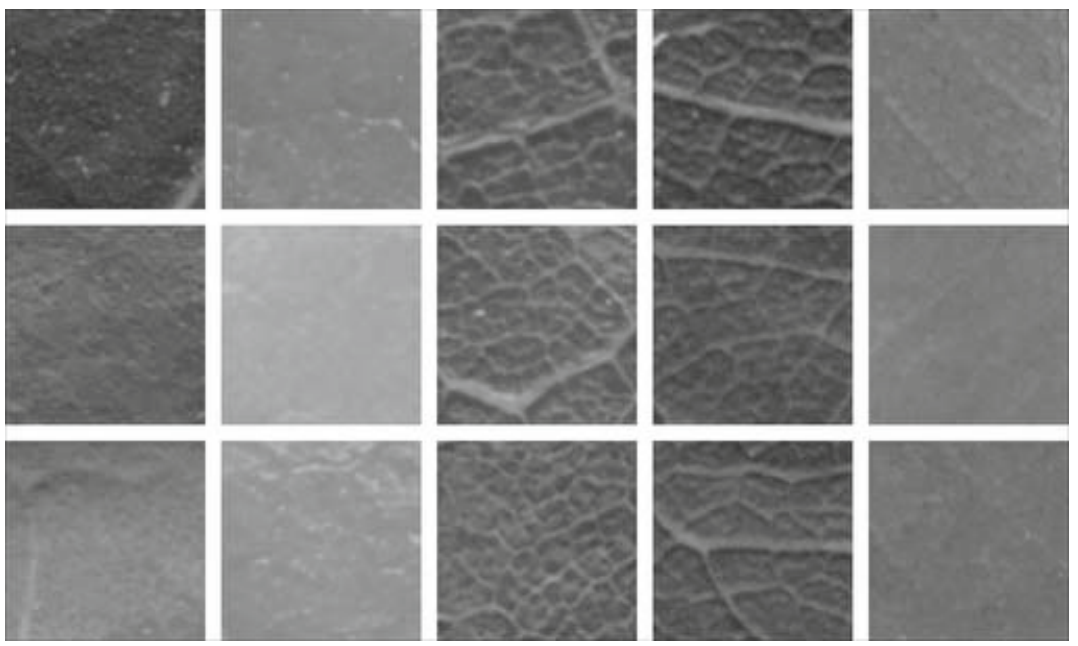

Fig. 4. Example of each texture class employed in the experiment. Example of texture variation in class (columns).

\section{$5 \quad$ Results}

Table11shows classification results yielded for the proposed approach. Multi-scale fractal dimension curves were computed from each texture pattern considered and best results were yielded considering dilation radius $r=7$. Derivative was computed using the Finite Difference method [30], thus resulting in a Multi-scale curve containing 50 descriptors.

Table 1. Classification performance of different texture descriptors

\begin{tabular}{|c|c|c|c|}
\hline Descriptor & $\begin{array}{c}\text { No of } \\
\text { Descriptors }\end{array}$ & $\begin{array}{c}\text { Samples correctly } \\
\text { classified }\end{array}$ & Success rate (\%) \\
\hline Gabor filters & 16 & 114 & 76.00 \\
Fourier descriptors & 63 & 94 & 62.66 \\
Co-occurrence matrices & 16 & 130 & 86.66 \\
M.S. Fractal Dimension & 50 & 135 & 90.00 \\
\hline
\end{tabular}

To provide a better performance evaluation, the proposed approach was compared with traditional texture analysis methods, such as Fourier descriptors [1], Co-occurrence matrices [4] and Gabor filters [8931]. A brief description of the compared methods is presented as follows:

Fourier descriptors: The bi-dimensional Fourier transform is applied over the texture sample. Shifting operation is performed over the Fourier spectrum values, so that, low frequencies are placed at the center of the spectrum. A total of 63 
descriptors is computed as being the energy of the spectrum values placed at a given radius distance from the center of the spectrum.

Co-occurrence matrices: This technique measures the joint probability distributions between pairs of pixels separated by a given distance and direction. Distances of 1 and 2 pixels with angles of $-45^{\circ}, 0^{\circ}, 45^{\circ}, 90^{\circ}$ were considered in this paper, as well as a non-symmetric version of the method. Many descriptors can be computed from co-occurrence matrices. For the experiments, we considered energy and entropy, totalising a set of 16 descriptors.

Gabor filters: This approach consists of convolving a 2-D Gabor filter over the original texture image. Each Gabor filter is a bi-dimensional gaussian function moduled with an oriented sinusoid in a determined frequency and direction. In this paper, we employed 16 filters ( 4 rotation filter and 4 scale filters), with lower and upper frequencies equal to 0.01 and 0.3, respectively. Energy was computed from each convolved image, so resulting in a set of 16 descriptors.

Results show that the proposed method is more robust, as it presents the highest success rate when compared with traditional texture analysis methods. This is mainly due to the great sensitiveness of the method to changes in the texture behavior. According to the dilation radius $r$ and the characteristics of the texture, the sphere produced by a pixel interferes on other spheres, and it disturbs the way the influence volume $S(r)$ increases. This disturbance in the influence volume allows to study pixels organization in the texture, as well as, its structural changes by complexity. Moreover, the use of Multi-scale Fractal dimension expands this analysis at different scales, so that, both micro and macro texture informations are considered and a better texture discrimination and classification are performed.

\section{Conclusion}

This paper presented a study of leaf texture classification based on complexity analysis. Leaf texture identification is a very difficult task due to the high similarity inter-classes and low similarity of intra-classes. Multi-scale Fractal Dimension computed from the Bouligand-Minkowski method was employed to output a curve that expresses the leaf texture complexity in terms of the spatial scale. These curves were evaluated in an experiment using linear discriminant analysis to classify a set of leaf texture previously selected. Results were compared with traditional texture analysis methods, and they show the great potential of the approach for natural texture analysis applications.

\section{Acknowledgments}

A.R.B. acknowledges support from FAPESP (2006/54367-9). O.M.B. acknowledges support from CNPq (306628/2007-4). 


\section{References}

1. Judd, W., Campbell, C., Kellog, E., Stevens, P.: Plant Systematics: A Phylogenetic Approach. Sinauer Associates, Massachusetts (1999)

2. Kurmann, M.H., Hemsley, A.R.: The Evolution of Plant Architecture. Royal Botanic Gardens, Kew (1999)

3. Hickey, L.R.: Classification of archictecture of dicotyledonous leaves. Amer. J. Bot. 60(1), 17-33 (1973)

4. Haralick, R.M.: Statistical and structural approaches to texture. Proc. IEEE 67(5), 786-804 (1979)

5. Murino, V., Ottonello, C., Pagnan, S.: Noisy texture classification: A higher-order statistics approach. Pattern Recognition 31(4), 383-393 (1998)

6. Shen, L., Bai, L.: A review on gabor wavelets for face recognition. Pattern Anal. Appl. 9(2-3), 273-292 (2006)

7. Bianconi, F., Fernández, A.: Evaluation of the effects of gabor filter parameters on texture classification. Pattern Recognition 40(12), 3325-3335 (2007)

8. Jain, A.K., Farrokhnia, F.: Unsupervised texture segmentation using Gabor filters. Pattern Recognition 24(12), 1167-1186 (1991)

9. Daugman, J., Downing, C.: Gabor wavelets for statistical pattern recognition. In: Arbib, M.A. (ed.) The Handbook of Brain Theory and Neural Networks, pp. 414-419. MIT Press, Cambridge (1995)

10. Manjunath, B.S., Ma, W.-Y.: Texture features for browsing and retrieval of image data. IEEE Trans. Pattern Anal. Mach. Intell 18(8), 837-842 (1996)

11. Azencott, R., Wang, J.-P., Younes, L.: Texture classification using windowed fourier filters. IEEE Trans. Pattern Anal. Mach. Intell 19(2), 148-153 (1997)

12. Bajcsy, R.K.: Computer identification of visual surfaces. Computer Graphics Image Processing 2, 118-130 (1973)

13. Sengür, A., Türkoglu, I., Ince, M.C.: Wavelet packet neural networks for texture classification. Expert Syst. Appl. 32(2), 527-533 (2007)

14. Unser, M.: Texture classification and segmentation using wavelet frames. IEEE Trans. Image Processing 4(11), 1549-1560 (1995)

15. Huang, P.W., Dai, S.K., Lin, P.L.: Texture image retrieval and image segmentation using composite sub-band gradient vectors. J. Visual Communication and Image Representation 17(5), 947-957 (2006)

16. Kaplan, L.M.: Extended fractal analysis for texture classification and segmentation. IEEE Transactions on Image Processing 8(11), 1572-1585 (1999)

17. Schroeder, M.: Fractals, Chaos, Power Laws: Minutes From an Infinite Paradise. W.H. Freeman, New York (1996)

18. Tricot, C.: Curves and Fractal Dimension. Springer, Heidelberg (1995)

19. Backes, A.R., Bruno, O.M.: A new approach to estimate fractal dimension of texture images. In: Elmoataz, A., Lezoray, O., Nouboud, F., Mammass, D. (eds.) ICISP 2008 2008. LNCS, vol. 5099, pp. 136-143. Springer, Heidelberg (2008)

20. Chen, Y.Q., Bi, G.: On texture classification using fractal dimension. IJPRAI 13(6), 929-943 (1999)

21. de O. Plotze, R., Falvo, M., Pádua, J.G., Bernacci, L.C., Vieira, M.L.C., Oliveira, G.C.X., Bruno, O.M.: Leaf shape analysis using the multiscale minkowski fractal dimension, a new morphometric method: a study with passiflora (passifloraceae). Canadian Journal of Botany 83(3), 287-301 (2005)

22. Li, J., Sun, C., Du, Q.: A new box-counting method for estimation of image fractal dimension. In: International Conference on Image Processing, pp. 3029-3032 (2006) 
23. da F. Costa, L., Cesar Jr., R.M.: Shape Analysis and Classification: Theory and Practice. CRC Press, Boca Raton (2000)

24. Carlin, M.: Measuring the complexity of non-fractal shapes by a fractal method. PRL: Pattern Recognition Letters 21(11), 1013-1017 (2000)

25. Bruno, O.M., de O. Plotze, R., Falvo, M., de Castro, M.: Fractal dimension applied to plant identification. Information Sciences 178, 2722-2733 (2008)

26. Emerson, C.W., Lam, N.N., Quattrochi, D.A.: Multi-scale fractal analysis of image texture and patterns. Photogrammetric Engineering and Remote Sensing 65(1), 51-62 (1999)

27. Gonzalez, R.C., Woods, R.E.: Digital Image Processing, 2nd edn. Prentic-Hall, New Jersey (2002)

28. Everitt, B.S., Dunn, G.: Applied Multivariate Analysis, 2nd edn. Arnold (2001)

29. Fukunaga, K.: Introduction to Statistical Pattern Recognition, 2nd edn. Academic Press, London (1990)

30. Smith, G.D.: Numerical Solution of Partial Differential Equations: Finite Difference Methods, 3rd edn., Oxford (1986)

31. Idrissa, M., Acheroy, M.: Texture classification using gabor filters. Pattern Recognition Letters 23(9), 1095-1102 (2002) 